\title{
2020 Market Analysis for 9th European Congress on Breast Cancer, Women's Health and Therapeutics
}

\section{Ibrahim Mashaal}

Senior Consultant, Department of Obstetrics and Gynecology, El-Mahalla General Hospital, Egypt, E-mail: ibrahim.mashaal@yahoo.com

The incidence on women diagnosed with Breast Cancer increases day after day. Out of 10 women, 3 are diagnosed with Breast Cancer and the rest are in the high-risk category, either already with associated diseases are at risk of acquiring it. With the everchanging food habits and the environmental changes, the no. of causes of Breast Cancer has been significantly risen. A study reveals that around 2200,000 women have been diagnosed with cancer or related diseases. And almost 0.5 million people die due to cancer around the world.

A strategic market analysis is of utmost important as it explains the case of incidence, the treatment methods available globally and cost of therapeutic systems. Of all the cancers related to women, breast cancer is revealed to be the second most cause of death and incidence, as revealed in the graph.

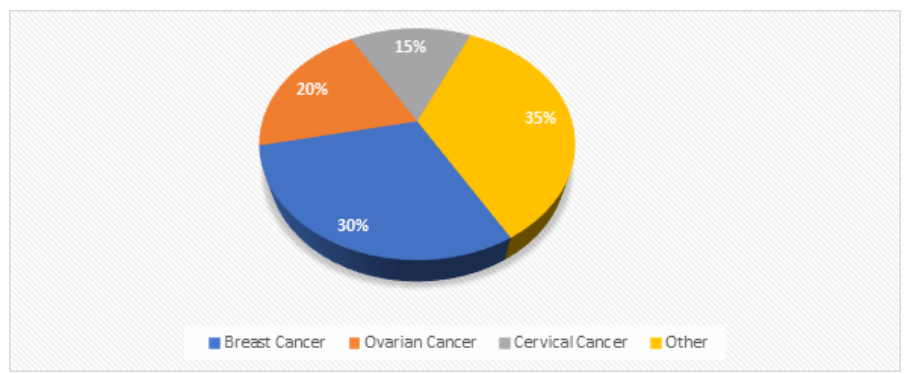

Diverse breast growth stages are there. On the off chance that breast growth is get distinguished in its beginning period the treatment turns out to be simple. In the beginning stage tumor is confined to the drain conduits, after some time it begins to spread in lymph hubs. Tumor at third stage is known as privately propelled breast disease, at this stage malignancy can be of any size as it spreads through lymph hubs to skin, chest and lymph hubs in under arms and in addition to inside mammary organs. Finally arrange breast growth do not stay to just to chest it invades into lungs, bones, liver and mind. Finally, it is characterized as tumor. Considering the phase of breast malignancy, treatment is given to understanding, so early discovery of breast disease is extremely important.

The recent developments in the field of therapeutics have proved to be curing breast cancer and other women related diseases for decades. Market study explains that, within the time of 2015-2020, the Compound Annual Growth Rate (CAGR) of the Therapeutics will see an increase up to $4.2 \%$ and the treatment therapeutics increase up to rate of US Dollars Nine hundred and Eighty Nine Million.

Therapeutics, has itself grown into various branches upon technological advancements and varies around the world. Chemotherapy, however, is widely used everywhere, accounting upon its cost effectiveness and efficient results, followed by Targeted therapy, immunotherapy, and hormonal therapy in the order.

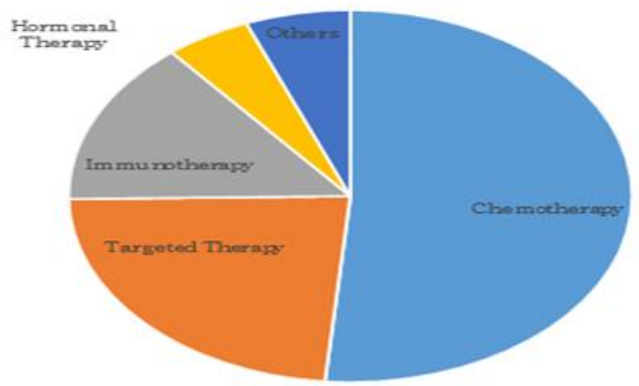

Treating menopausal symptoms with estrogen and progestin together is known as estrogen-progestin therapy or combined hormone therapy. Hormonal replacement therapy (HRT) has a higher risk of being diagnosed with breast cancer. Before the link between HRT use and breast cancer risk was established, many postmenopausal women took HRT for many years to ease menopausal symptoms (hot flashes, fatigue) and to reduce bone loss. Since 2002, when research linked HRT and risk, the number of women taking HRT has dropped dramatically. Still, many women continue to use HRT to handle bothersome menopausal symptoms.

Stem-cell therapy is the use of stem cells to treat or prevent a disease or condition. In a typical stem cell transplant for cancer very high doses of chemo are used, sometimes along with radiation therapy, to try to kill all the cancer cells. This treatment also kills the stem cells in the bone marrow. Soon after treatment, stem cells are given to replace those that were destroyed. These stem cells are given into a vein, much like a blood transfusion. Over time they settle in the bone marrow and begin to grow and make healthy blood cells. This process is called engraftment.

The below graph explains the incidence of breast cancer, in the top European countries in the year of 2018. The graph explains an age-standardised report, a measure of incidence of breast cancer at a standardised age. We ca infer from the graph that almost equal risk is been observed in all the designated 9 nations, namely, Belgium, Luxembourg, Netherland, France, New Caledonia, Lebanon, Australia, UK, Italy. We hence can conclude; the distribution of the disease is uniform.

Reducing the intensity of Bone disease in Women associates with accurate diagnosis when symptoms are visible, incorporating appropriate treatment and follow-ups, Diagnostic check-ups along with Laboratory tests help women to know the bone health at the earliest. Genetic and External factors can also affect the Bone Health i.e., Osteogenesis Imperfecta and Endocrine disorders.

Aging is a natural phenomenon but healthy aging in women 
depends on the many risk factors and one major would be Breast Cancer -it is a leading cause of death, autoimmune diseases etc, the treatment methods are discussed and for a successful aging.

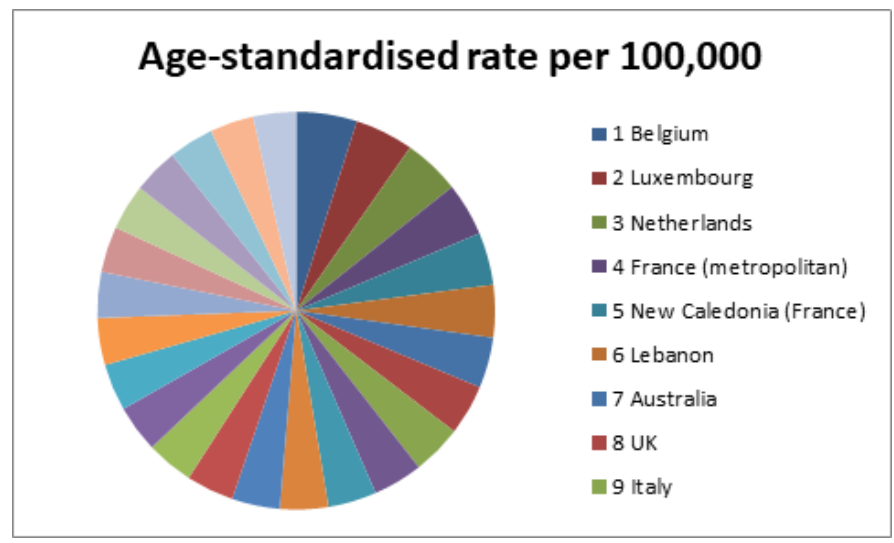

Clinical Trials on Breast Cancer have found to be optional, individuals enroll under the clinical trials as treatments too, pre-clinical trials are done on mice to find the adverse effects. Individual can voluntarily participate in certain clinical trials done in Breast Cancer. Pre-clinical trials help in understanding whether the treatment is safe or not. Side effects are there for all treatments done in breast cancer and can be sorted out during clinical trials. Research in breast cancer can be related to clinical trials as well. Currently researches of Breast cancer is happening in genetic level. Healthcare experts believe there are faulty genes affecting the breast in adverse way. Doctors are learning by evaluating families and generations who have a fault in the certain genes and exposure to breast cancer.

Rehabilitation after the cancer treatment might sound the struggle to survive. The true fact is life after the treatment especially for a woman after the breast cancer there will be new choices to make and even new opportunities. Altering the food habits to daily exercise are certain things which can be and should be implemented in daily life, so that there won't be any recurrence of breast cancer.

Hair development begins once more. Physical vitality and working can likewise be getting influenced after the breast disease treatment. Level of estrogen may likewise get brought down after the treatment which prompts the diminishing in bone thickness. Bone thickness can be expanded also repeat of breast growth can be counteracted with the assistance of exercise. 